\title{
Image Reconstruction using Fast Inverse Half tone and Huffman Coding Technique
}

\author{
Dr. H.B. Kekre \\ Senior Professor, Department of \\ Computer Science, \\ Mukesh Patel School of Technology \\ Management and Engineering \\ Mumbai, India
}

\author{
Dr. Tanuja K. Sarode \\ Professor, Department of Computer \\ Science, TSEC, Bandra, Mumbai \\ University, Mumbai
}

\author{
Ankit Lahoti, Gauri Sawant \\ Undergraduate \\ Mukesh Patel School of Technology \\ Management and Engineering \\ Mumbai, India
}

\author{
Sanjay R. Sange \\ Assistant Professor and Research \\ Scholar, Department of Information \\ Technology, PhD scholar student \\ Mukesh Patel School of Technology \\ Management and Engineering
}

Mumbai, India

\begin{abstract}
Transmission of audio-video data over internet applications like Multimedia is increasing with fast pace. Biometric, Content Based Image Retrieval (CBIR), CCTV footage applications require huge storage of images in database. For such applications this combination of half tone with Huffman coding is useful. Half toning is lossy technique used in printing industry where binary image is required. Objective of achieving higher Compression Ratio by combining lossy half tone and lossless Modified Huffman coding techniques is used. Apart from standard operator like Floyd-Steinberg and Jarvis operators, Small and South-East operator are used. Halftone and Huffman coding technique is implemented on 10 different color images of size $512 \times 512$. For measurement of image quality, Mean Square Error (MSE) and Peak Signal-to-Noise Ratio (PSNR) and Structure Similarity Index (SSIM) are used. This hybrid technique can use for low bit rate video data transmission and mass image storage.
\end{abstract}

\section{Keywords}

Halftone, error diffusion, gradient estimation, concatenation, Structure Similarity Index (SSIM), Huffman coding

\section{INTRODUCTION}

For reconstruction of image Fast Inverse half toning algorithm is used [1], [2], [3] and [4]. For reconstruction of image, many iterations are required that increases computational complexity and memory space [2], [3] and [4]. In this paper, Fast Inverse half toning algorithm in which $7 \times 7$ matrix is used as multi-scale gradient estimation for edge detection while other technique requires heavy use of floating point operations. This algorithm takes single pass along both the axes for filtration instead of multiple iterations. In this algorithm, most of the processes require integer additions and seven rows are kept in memory at a time. After half toning process quantization process is applied to get binary image in which error diffusion introduces noise. Reconstruction of image from such half tone image without loss is impossible. Inverse image is blurred image because of Finite Impulse Response (FIR) Low pass Filter. Halftone technique is

used in printing media where image is in binary form. Halftone technique converts continuous tone image into half tone image. Floyd-Steinberg and Jarvis half toning operators preserve the artifacts in the image [3], [5]. Various halftone operators are explained in [5] and [6]. For further compression of image data, lossless Run Length Encoding [7], Huffman coding technique with different approaches is used in paper [8] to [12].

In this paper, section 2 is the implementation of Halftone and Huffman coding Algorithm. Section 3 gives the implementation of Fast Inverse Half toning algorithm. In section 4 introduces Structure Similarity Index (SSIM), the measuring parameter for quality measurement between reconstructed and original image. Section 5 gives the discussion about various half toning operators and significance of measuring parameters. Section 6 shows the experimental results in the form of images and measuring parameters like Mean Square Error (MSE), SSIM and Peak Signal-to-Noise Ratio (PSNR) are given in Table-1. Same section gives parameter comparison in graphical format and brief discussion about the same. Conclusion and future scope of the paper is explained in section 7. Section 8 is the paper references. This is the extension of paper [1] in which reconstruction of image using Fast Inverse Half toning algorithm is explained.

\section{HALF TONE AND HUFFMAN CODING}

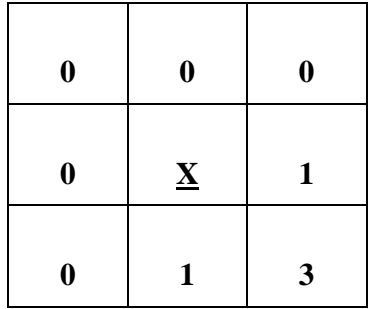

Fig 1.a): Small Operator

\begin{tabular}{|c|c|c|}
\hline 0 & 0 & 0 \\
\hline $\mathbf{0}$ & $\underline{\mathbf{X}}$ & $\mathbf{7}$ \\
\hline $\mathbf{3}$ & $\mathbf{5}$ & $\mathbf{1}$ \\
\hline
\end{tabular}

Fig 1.b): Floyd-Steinberg Operator 


\begin{tabular}{|l|l|l|l|l|}
\hline 0 & 0 & 0 & 0 & 0 \\
\hline 0 & 0 & 0 & 0 & 0 \\
\hline 0 & 0 & $\underline{x}$ & 7 & 5 \\
\hline 3 & 5 & 7 & 5 & 3 \\
\hline 1 & 3 & 5 & 3 & 1 \\
\hline
\end{tabular}

Fig 1.c): Jarvis Operator

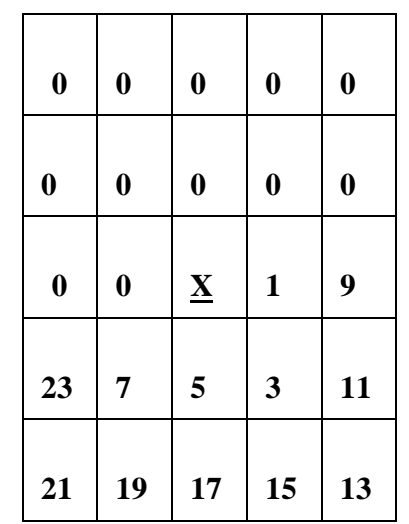

Fig 1. d): South-East Operator

Each plane of continuous tone image is having 0 to 255 gray levels. This entire range is converted into binary values i.e. 0 and 1 , hence this is lossy technique. Huffman coding has unique codes, from which image can be decoded without loss.

Fig1. a) - fig1. d) Shows different half toning operators. These operators are used and obtained satisfactory experimental results. $\underline{\mathbf{X}}$ shown in all operators is the central pixel. The half toning operator is convolved with the original image and quantization process is applied so as to get half tone single bit image [1], [7]. Floyd-Steinberg and Jarvis half toning are the standard operators and the Small and South-East operator are presented in paper [7]. During neighborhood processing Small, Floyd-Steinberg, Jarvis and South-East half toning operators require 3 tap, 4 tap, 12 tap and 12 tap effectively 1 tap, 3 tap, 10 tap and 11 tap operation respectively. Half toning process converts 8-bit gray scale image into 1-bit image and gives 8:1 compression ratio, hence it is a lossy technique. During quantization process quantization error is introduced is called as blue noise [1], [7]. As shown in fig2 color image is split into three primary colors R-G-B individual plane. After the half tone process, sampling and quantization process is applied on each individual plane and pixel will be represented by single bit instead of 8 bit per pixel.

Modified Huffman coding is used for higher compression ratio [7] where less than one bit per pixel is required. The compression ratio between half tone image and Huffman coded image is 2.99 irrespective of the input image. The coded sequence of the halftone image data has been considered in group of 16 bits in size to minimize the network resources and storage required. Each of the 16 bits groups is converted into their decimal equivalent on which Huffman coding is applied.

\section{FAST INVERSE HALFTONING ALGORITHM}

Different types of error diffusion half tone operators as well as block diagram for image reconstruction are expressed in paper [1] and [2]. With the help of multi-scale gradient estimation both edges are traced. As it require minimum multiplication, division and negligible floating point operations that lowest computational complexity and memory usage [1].

Fig 2 shows the block diagram for reconstruction of image from Huffman decoded image. Huffman coding is the lossless

technique; hence it is the color half tone image and is split into three primary R-G-B colors. From each plane vertical and horizontal edges are traces using gradient estimation. Control function is defined in [1] that calculates the cut-off frequency for FIR separable Low Pass filter. Because of Low Pass filter image looks little bit blurry. Color 24-bit image is reproduced using concatenation technique.

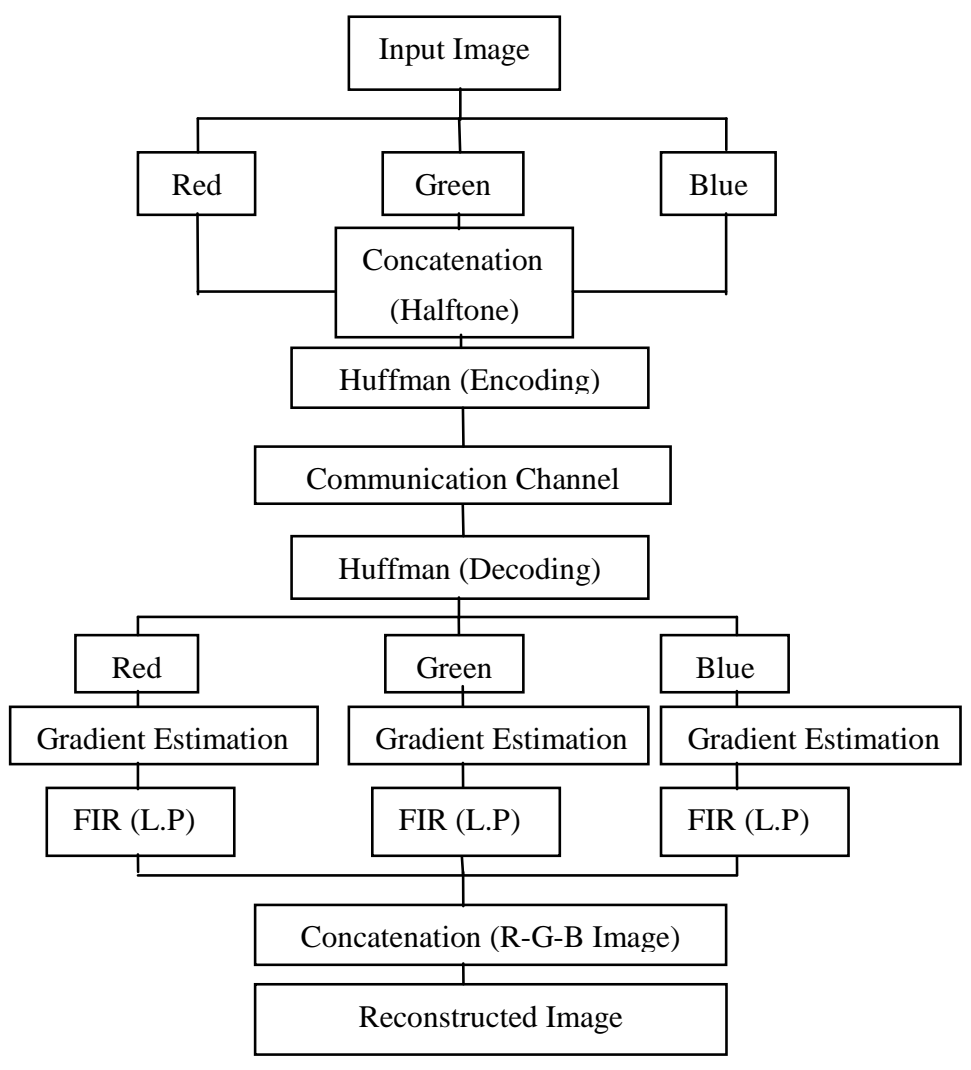

Fig 2: Block diagram

\section{Structure Similarity Index (SSIM)}

The Structural Similarity (SSIM) index is a measuring parameter between original image and its processed image. As shown in equation (1), image $\mathrm{x}$ is considered as input image and image $\mathrm{y}$ is inverse image. This measuring parameter gives structure wise similarity between two images. Block of size $8 \times 8$ is considered for both image $\mathrm{x}$ and image $\mathrm{y}$. SSIM is the quality measure on the degradation of structural information and for calculation of Structural Similarity Index the measurement system is presented in [13]. Some quality measuring approaches are presented in [14]-[15].

$$
\operatorname{SSIM}(x, y)=\frac{\left(2 \mu_{x} \mu_{y}+c 1\right)\left(2 \operatorname{cov}_{x y}+c 2\right)}{\left(\mu_{x 2}+\mu_{y 2}+c 1\right)\left(\sigma_{x}{ }^{2}+\mu_{y}{ }^{2}+c 2\right)}
$$

Where, 1) $\boldsymbol{\mu}_{\mathbf{x}}$ is the average of image $x$.
2) $\boldsymbol{\mu}_{\mathrm{y}}$ is the average of image $y$.
3) $\boldsymbol{\sigma}_{x}{ }^{2}$ is the variance of image $x$.
4) $\sigma_{y}{ }^{2}$ is the variance of image $y$ 
5) $\operatorname{cov}_{\mathbf{x y}}$ is the co-variance between two images $\mathrm{x}$ and $\mathrm{y}$.

6) $\mathbf{C 1}=\left(\mathbf{k}_{1} \cdot \mathbf{L}\right)^{2}$ and $\mathbf{C}_{2}=\left(\mathbf{k}_{2} \cdot \mathbf{L}\right)^{\mathbf{2}}$ are the two variables to stabilize the division with small value denominator, where $\mathrm{L}$ is the dynamic range of pixel values i.e. $\mathbf{2}^{\mathbf{n}}$.

7) $\mathbf{k}_{\mathbf{1}}=\mathbf{0 . 0 1}$ and $\mathbf{k}_{\mathbf{2}}=\mathbf{0 . 0 3}$ by default

The range for SSIM is -1 to +1 . For both the identical images SSIM is +1 and SSIM is -1 for worse output image as compared to input image. Structural dissimilarity (DSSIM) is a distance metric derived from SSIM.

$$
\operatorname{DSSIM}(x, y)=\frac{1}{1-\operatorname{SSIM}(x, y)}
$$

\section{DISCUSSION}

The overall objective of this paper is to achieve higher compression ratio to real-time video data streaming. As mentioned in section2 effective number of taps determines the computational complexity. So, Small operator gives the single tap operation with acceptable image quality. SSIM is designed to improve on traditional methods like Peak Signal to Noise Ratio (PSNR) and Mean Square Error (MSE), which have proved to be inconsistent with human eye perception. Even though MSE is same in two different cases but appearance point of view both images are quite different. From human visual system point of view SSIM gives the accurate result. Typically it is calculated on window sizes of $8 \times 8$. Window size can be changed to reduce the complexity.

Image shown in fig 3.a) is the sample image of Rohit as an original image. Small half toning operator is used to convert original image into halftone image shown in fig 3.b), fig 3.c), and fig 3.d) are the Huffman decoded image and inverse image respectively. In the same way each code is unique and this helps in decoding the coded sequence easily. The decoded sequence is then arranged in a matrix of the size of the original image and thus the Huffman decoded image is obtained, which is identical to that of halftone image. Huffman coding technique is lossless but useful for higher compression.

\section{EXPERIMENTAL RESULTS}

The algorithm discussed above is implemented using MATLAB 2008 b on P8600 @ 2.40GHz, 2.92 GB RAM. To test the performance of this algorithm four color images belonging to different classes of size $512 \times 512 \times 3$ are used.

Table-1 shows the result of 10 sample images of different categories. Fig 4.a), 4.b) and 4.c) are the graphical presentation of sample images with respect to MSE, SSIM, and PSNR respectively. Fig 4.a) shows that MSE is almost same for all half toning operators. Appearance point of view quality of inverse image from Floyd-Steinberg and Jarvis half toning operators is better. With single tap operation i.e. lowest computation inverse image generated from Small operator is also with acceptable quality. Half tone image produced by Floyd-Steinberg is very good retains pictorial details as compared to other half tone operators. Jarvis half toning operator preserves edges and artifacts. Whereas the inverse image of South-East half tone operator looks patchy with some shadow effects. Fig 3 b) is the half tone image from Small operator and fig $3 \mathrm{c}$ ) is the Huffman decoded image from it. Fig 3 e) is the half tone image from Floyd-Steinberg operator and fig $3 \mathrm{f}$ ) is the Huffman decoded image from it. Fig $3 \mathrm{~h}$ ) is the half tone image from Jarvis operator and fig 3 i) show the Huffman decoded image from it. Fig $3 \mathrm{k}$ ) is the half tone image from South-East operator and fig 3 l) show the Huffman decoded image from it. Fig 3 g), and fig $3 \mathrm{j}$ ) are the reconstructed images from Floyd-Steinberg and Jarvis operator are of fine image quality as compared to fig $3 \mathrm{~d}$ ) and fig $3 \mathrm{~m}$ ) of Small and South-East operator. Fig $3 \mathrm{~d}$ ) is little-bit course and fig $3 \mathrm{~m}$ ) looks quite patchy image. From computation point of view Small operator require lowest number of computations as explained in section 2. Table 1 shows SSIM value approaches to zero means from structure point of view reconstructed image is moderate with respect to input image.

\section{CONCLUSION AND FUTURE SCOPE}

In this paper, we have used standard half toning operates along with proposed Small and South-East operators. Small and South-East operator's give almost same result as compared Floyd-Steinberg and Jarvis operator in terms of measuring parameter. It means reconstructed image quality matches with input image considerably. In Fast Inverse half tone algorithm FIR Low Pass filter is used. The scope to this paper is to develop half toning operators and design of filter to minimize the blurring effect. In printing media it is required to store huge number of images as well as transmission of images, videos worldwide. In such kind of applications this hybrid technique can be utilized. From result the image quality is acceptable and can be used for low bit rate video data transmission, the same work is under process. The same technique can be used for storage of CCTV footage in hybrid encoded form. Stream of video can be cut into number of frames using Virtual -dub or Frame-Shot software. For video conferencing high speed communication is required and that can be achieved by skipping some of the frames based on threshold.

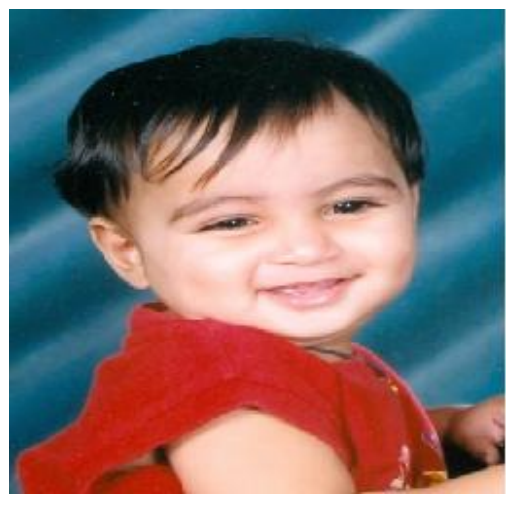

Fig 3.a): Original Image: Rohit 


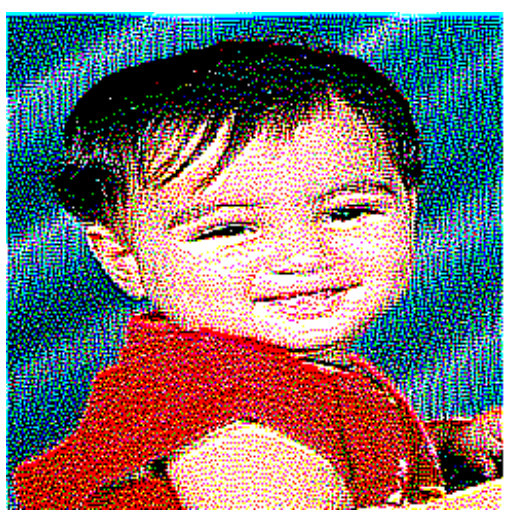

Fig 3.b): Small Half tone Image

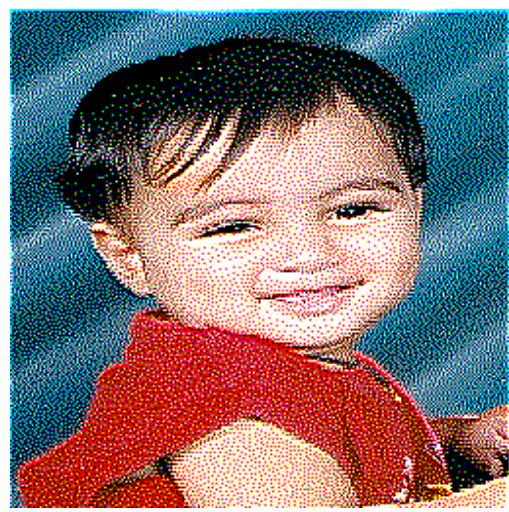

Fig 3.e): Floyd-Steinberg Half tone Image

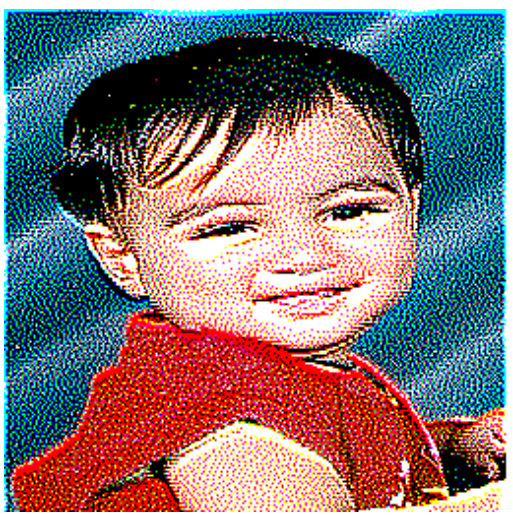

Fig 3.h): Jarvis Half tone Image

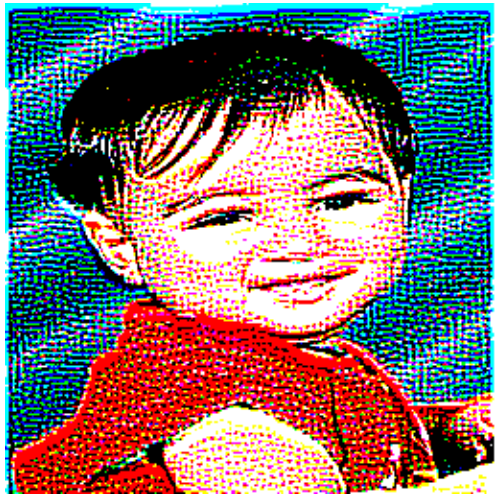

Fig 3.k): South-East Half tone Image

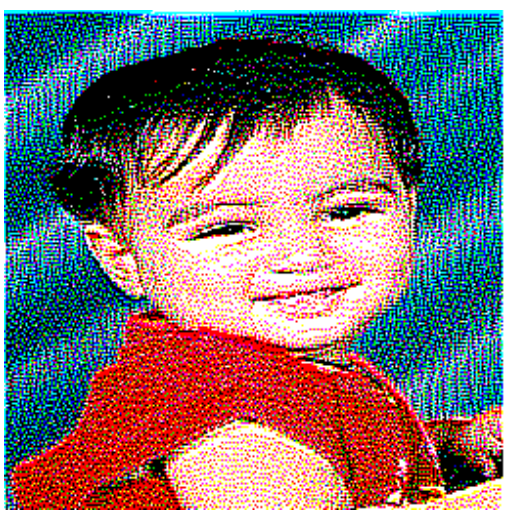

Fig 3.c): Small - Huffman Decoded Image

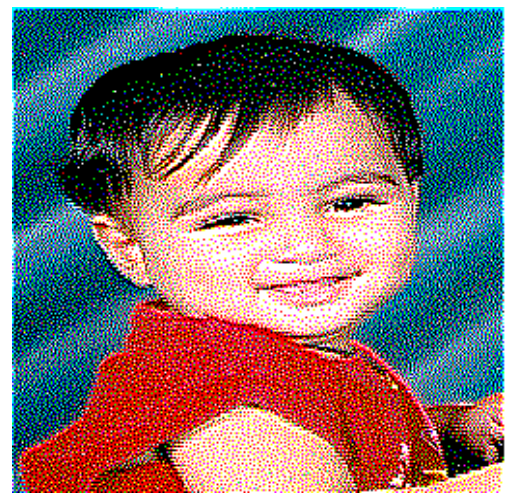

Fig 3.f): Floyd-Steinberg- Huffman Decoded Image

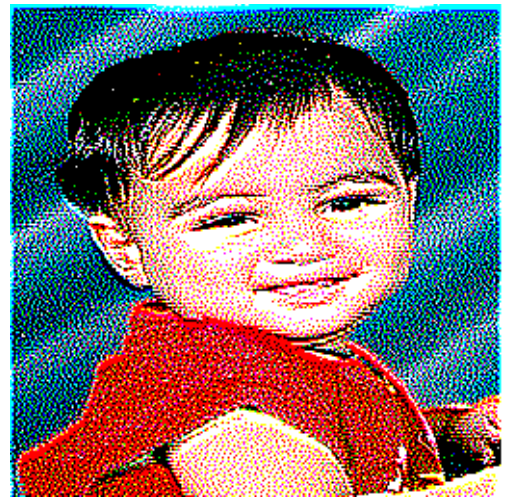

Fig 3.i): Jarvis - Huffman Decoded Image

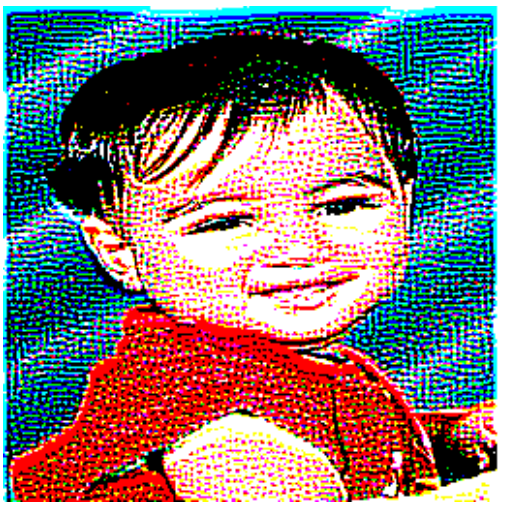

Fig 3.1): South-East - Huffman Decoded Image

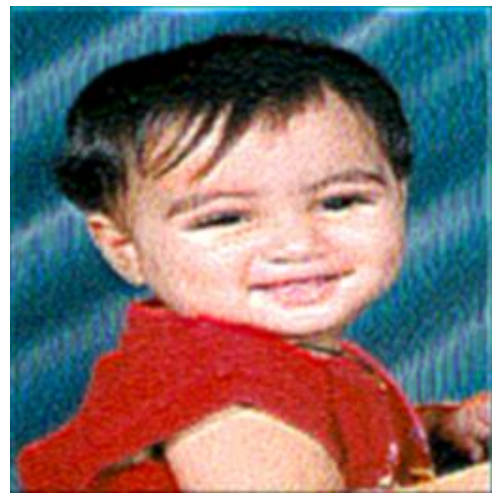

Fig 3.d): Reconstructed image

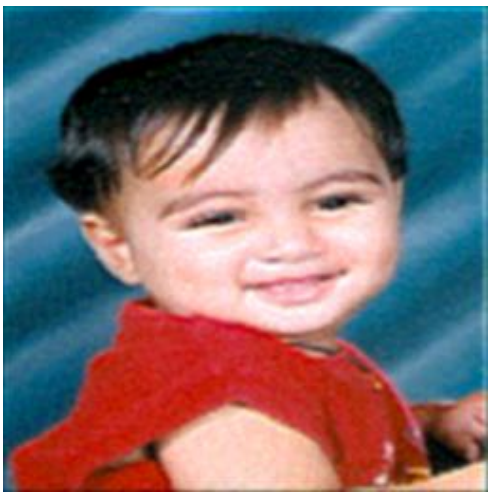

Fig 3.g): Reconstructed image

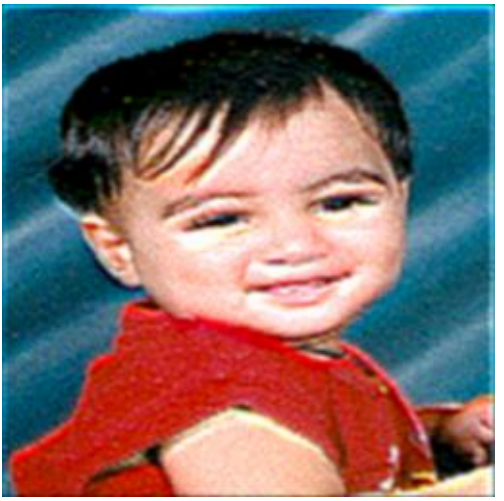

Fig 3.j): Reconstructed image

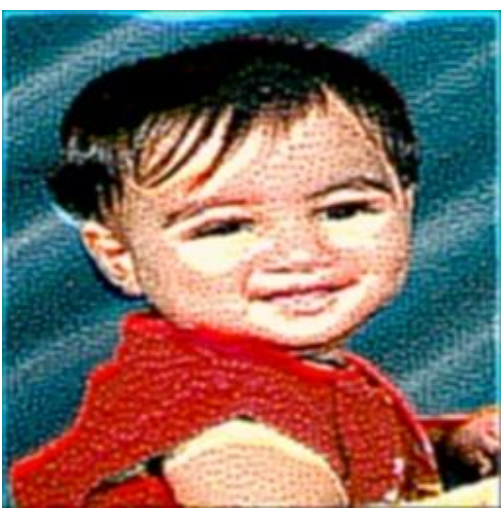

Fig 3.m): Reconstructed image 
Table1. Measuring parameters like Mean Square Error (MSE), PSNR and Structure Similarity Index (SSIM) used for different images and half tone operators.

\begin{tabular}{|c|c|c|c|c|c|}
\hline Image & Parameter & $\begin{array}{l}\text { Floyd-Stenberg } \\
\text { Operator }\end{array}$ & Jarvis Operator & Small Operator & South-East Operator \\
\hline Baboon & $\begin{array}{l}\text { SSIM } \\
\text { MSE } \\
\text { PSNR }\end{array}$ & $\begin{array}{l}8.8218 \mathrm{e}-005 \\
17715 \\
42.4834\end{array}$ & $\begin{array}{l}8.5575 \mathrm{e}-005 \\
17719 \\
42.4843\end{array}$ & $\begin{array}{l}8.6894 \mathrm{e}-005 \\
17717 \\
42.4839\end{array}$ & $\begin{array}{l}8.7714 \mathrm{e}-005 \\
17718 \\
42.4842\end{array}$ \\
\hline Cloud & $\begin{array}{l}\text { SSIM } \\
\text { MSE } \\
\text { PSNR }\end{array}$ & $\begin{array}{l}7.2531 \mathrm{e}-005 \\
15027 \\
41.7686\end{array}$ & $\begin{array}{l}6.7736 \mathrm{e}-005 \\
15032 \\
41.7701\end{array}$ & $\begin{array}{l}7.0133 \mathrm{e}-005 \\
15029 \\
41.7694\end{array}$ & $\begin{array}{l}7.8740 \mathrm{e}-005 \\
15023 \\
41.7675\end{array}$ \\
\hline Lata & $\begin{array}{l}\text { SSIM } \\
\text { MSE } \\
\text { PSNR }\end{array}$ & $\begin{array}{l}8.2293 \mathrm{e}-005 \\
16461 \\
42.1646\end{array}$ & $\begin{array}{l}8.2293 e-005 \\
16461 \\
42.1646\end{array}$ & $\begin{array}{l}8.0988 \mathrm{e}-005 \\
16462 \\
42.1648\end{array}$ & $\begin{array}{l}8.2793 e-005 \\
16460 \\
42.1644\end{array}$ \\
\hline LenaColor & $\begin{array}{l}\text { SSIM } \\
\text { MSE } \\
\text { PSNR }\end{array}$ & $\begin{array}{l}7.8322 \mathrm{e}-005 \\
18897 \\
42.7639\end{array}$ & $\begin{array}{l}8.0062 \mathrm{e}-005 \\
18899 \\
42.7643\end{array}$ & $\begin{array}{l}7.8723 \mathrm{e}-005 \\
18898 \\
42.7642\end{array}$ & $\begin{array}{l}8.2200 \mathrm{e}-005 \\
18898 \\
42.7642\end{array}$ \\
\hline Pepper & $\begin{array}{l}\text { SSIM } \\
\text { MSE } \\
\text { PSNR }\end{array}$ & $\begin{array}{l}6.6770 \mathrm{e}-005 \\
29247 \\
44.6608\end{array}$ & $\begin{array}{l}6.8110 \mathrm{e}-005 \\
29244 \\
44.6604\end{array}$ & $\begin{array}{l}6.7159 \mathrm{e}-005 \\
29246 \\
44.6607\end{array}$ & $\begin{array}{l}6.9335 \mathrm{e}-005 \\
29242 \\
44.6601\end{array}$ \\
\hline Rock & $\begin{array}{l}\text { SSIM } \\
\text { MSE } \\
\text { PSNR }\end{array}$ & $\begin{array}{l}8.1580 \mathrm{e}-005 \\
9690 \\
39.8633\end{array}$ & $\begin{array}{l}8.5367 e-005 \\
9688 \\
39.8627\end{array}$ & $\begin{array}{l}8.3284 \mathrm{e}-005 \\
9689 \\
39.8629\end{array}$ & $\begin{array}{l}9.1342 \mathrm{e}-005 \\
9685 \\
39.8613\end{array}$ \\
\hline Rohit4 & $\begin{array}{l}\text { SSIM } \\
\text { MSE } \\
\text { PSNR }\end{array}$ & $\begin{array}{l}6.7379 \mathrm{e}-005 \\
22950 \\
43.6078\end{array}$ & $\begin{array}{l}6.8466 \mathrm{e}-005 \\
22949 \\
43.6076\end{array}$ & $\begin{array}{l}6.5358 \mathrm{e}-005 \\
22953 \\
43.6084\end{array}$ & $\begin{array}{l}6.9780 \mathrm{e}-005 \\
22947 \\
43.6073\end{array}$ \\
\hline Rohit11 & $\begin{array}{l}\text { SSIM } \\
\text { MSE } \\
\text { PSNR }\end{array}$ & $\begin{array}{l}6.9905 e-005 \\
23734 \\
43.7538\end{array}$ & $\begin{array}{l}7.0903 \mathrm{e}-005 \\
23733 \\
43.7536\end{array}$ & $\begin{array}{l}6.9487 \mathrm{e}-005 \\
23735 \\
43.7539\end{array}$ & $\begin{array}{l}6.7828 \mathrm{e}-005 \\
23739 \\
43.7545\end{array}$ \\
\hline RohitKrishna & $\begin{array}{l}\text { SSIM } \\
\text { MSE } \\
\text { PSNR }\end{array}$ & $\begin{array}{l}6.4643 e-005 \\
23500 \\
43.7107\end{array}$ & $\begin{array}{l}6.6545 \mathrm{e}-005 \\
23499 \\
43.7105\end{array}$ & $\begin{array}{l}6.4804 e-005 \\
23501 \\
43.7108\end{array}$ & $\begin{array}{l}6.7976 \mathrm{e}-005 \\
23498 \\
43.7103\end{array}$ \\
\hline RohitSmile & $\begin{array}{l}\text { SSIM } \\
\text { MSE } \\
\text { PSNR }\end{array}$ & $\begin{array}{l}7.5209 \mathrm{e}-005 \\
17830 \\
42.5116\end{array}$ & $\begin{array}{l}7.5234 \mathrm{e}-005 \\
17831 \\
42.5118\end{array}$ & $\begin{array}{l}7.4972 \mathrm{e}-005 \\
17831 \\
42.5118\end{array}$ & $\begin{array}{l}7.4494 \mathrm{e}-005 \\
17833 \\
42.5121\end{array}$ \\
\hline
\end{tabular}




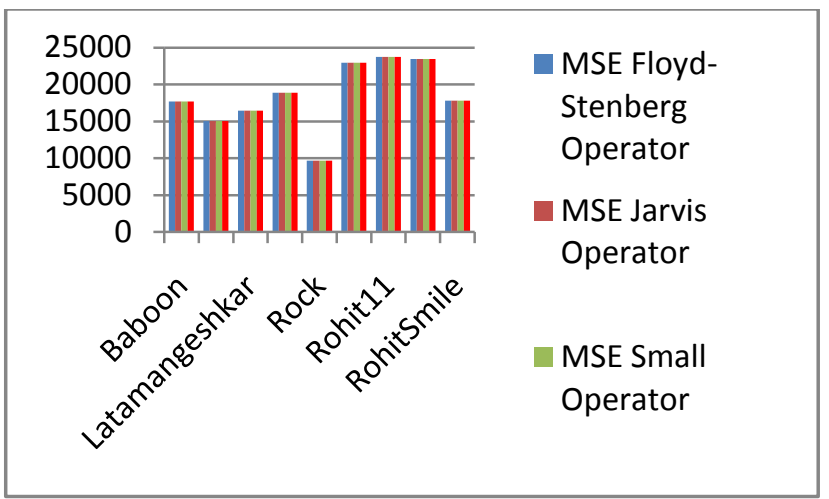

Fig 4.a): Different images versus MSE with different operators

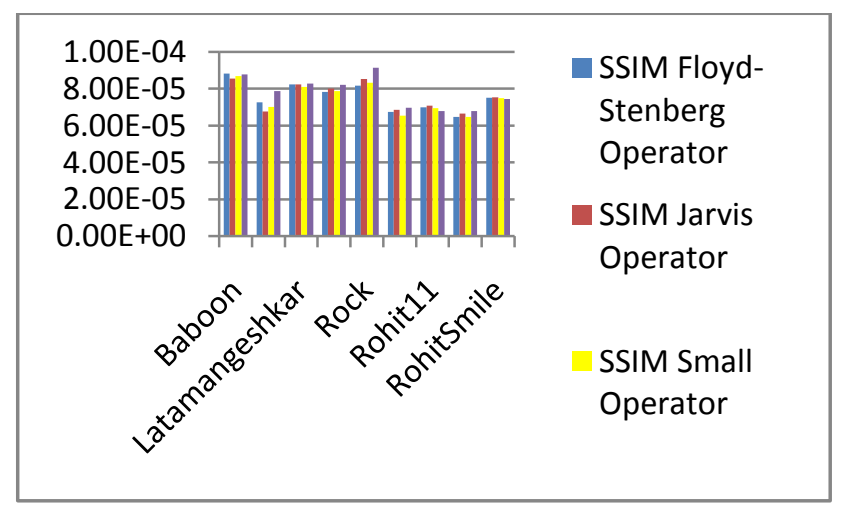

Fig 4.b): Different images versus SSIM with different operators

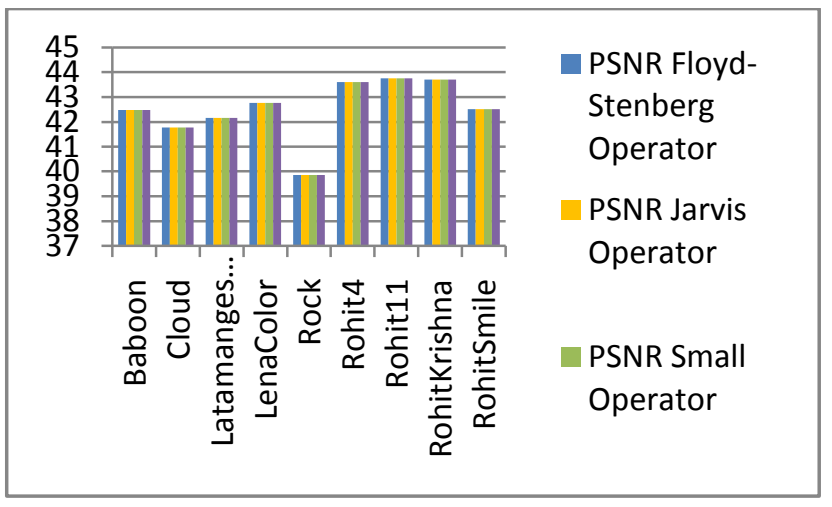

Fig 4.c): Different images versus PSNR with different operators

\section{REFERENCES}

[1] Sanjay R. Sange, 2009 Restoration of Color Halftone image by using Fast Inverse Half toning Algorithm, in 2009 International Conference on Advances in Recent Technologies in Communication and Computing, 978-07695-3845-7/09 \$25.00 (C) 2009 IEEE, DOI 10.1109/ARTCom.2009.36, pg .650 -655,Oct.(2009)

[2] Hein, S., Zakhor, A, 1995Halftone to continuous-tone conversion of Error-diffusion coded images, IEEE Trans. Images Processing, vol.4, pp.208--216, Feb. (1995).
[3] Floyd, R. W., Steinberg, 1976, An adaptive algorithm for spatial grayscale, Proc. SID, vol. 17/2, pg. 75--77, (1976)

[4] Wong P., 1995 Inverse half toning and Kernel estimation for error diffusion, IEEE Trans. Image Processing, vol.4, pp.. 486--498, Apr (1995)

[5] Sange, S., 2009 A Survey on, Black and White and Color Half toning Techniques, SVKM's NMIMS University, MPSTME, Journal of science, Engineering \& Technology Management, ISSN: 0975-525X Techno-Path" Vol.1 No.2-May (2009), pg. no. 7--17

[6] Kite, T. D., Evans, B. L., Bovik, A. C., 2000 Modeling and Quality Assessment of Half toning by Error Diffusion, IEEE Transaction on Image Processing, vol.9.No.5, May (2000).

[7] Sange, S. R., 2010 Image data compression using new Halftoning operators and Run Length Encoding. In: $1^{\text {st }}$ International Conference Thinkquest2010. Published in Springer Explorer and Springer CS Digital Library, pg224-230, Feb (2010)

[8] H. B. Kekre, Sanjay R. Sange, Gauri S. Sawant, and Ankit A. Lahoty, 2011, Image Compression Using Half toning and Huffman Coding, ICTSM 2011, CCIS 145, pp.221226, 2011. (C) Springer-Verlag Berlin Heidelberg 2011

[9] Pujar, J. H., Kadlaskar, L. M. 2005 - 2010, A New Lossless Method of Image Compression and Decompression using Huffman Coding Techniques, Journal of Theoretical and Applied Information Technology, (2005 - 2010) JATIT.

[10] Saravanan, C. Ponalagusamy, R. 2005 - 2010, Lossless Grey-scale Image Compression using Source Symbols Reduction and Huffman Coding, International Journal of Image Processing (IJIP), Volume (3): Issue (5), (2005 2010) JATIT.

[11] Aggarwal M. Narayan A. 2000, An Efficient Huffman Decoding", in (2000) International Conference on Image Processing, IEEE Proceedings, pg.936-939 vol.1

[12] Tehranipour, M. H. Nourani, 2010, Mixed RL-Huffman encoding for power reduction and data compression in scan test, In: Intelligent Information Technology and Security Informatics (IITSI), 2010 Third International Symposium on 2-4 April (2010).

[13] Zhou Wang, Alan Conarad Bovik, Hamid Rahim Sheikh, 2004, Image Quality Assessment: From Error Visibility to Structural Similarity IEEE Transactions on Image Processing, Vol.13, No. 4, April 2004.

[14] Z. Wang, Dec. 2001, Rate scalable Foveated image and video communications, Ph.D. dissertation, Dept. Elect. Comput. Eng., Univ. Texas at Austin, Austin, TX, Dec. 2001.

[15] Zhou Wang, A. C. Bovik,, Mar. 2002, A Universal image quality index, IEEE Signal Processing Letters, vol.9, pp. 81-84, Mar. 2002. 


\section{AUTHOR BIOGRAPHIES}

Dr. H. B. Kekre has received B.E. (Hons.) in Telecomm. Engg. from Jabalpur University in 1958, M.Tech (Industrial Electronics) from IIT Bombay in 1960, M.S.Engg. (Electrical Engg.) from University of Ottawa in 1965 and Ph.D. (System Identification) from IIT Bombay in 1970. He has worked Over 35 years as Faculty of Electrical Engineering and then HOD Computer Science and Engg. at IIT Bombay. For last 13 years worked as a Professor in Department of Computer Engg. at Thadomal Shahani Engineering College, Mumbai. He is currently Senior Professor working with Mukesh Patel School of Technology Management and Engineering, SVKM's NMIMS University, Vile Parle(w), Mumbai, INDIA. He has guided 17 Ph.D.s, 150 M.E./M.Tech Projects and several B.E./B.Tech Projects. His areas of interest are Digital Signal processing, Image Processing and Computer Networks. He has more than 350 papers in National / International Conferences / Journals to his credit. Recently 12 students working under his guidance have received best paper awards. Five of his students have been awarded Ph. D. of NMIMS University. Currently he is guiding ten $\mathrm{Ph} . \mathrm{D}$. students. He is member of ISTE and IETE.

Dr. Tanuja K. Sarode has received M.E.(Computer Engineering) degree from Mumbai University in 2004, Ph.D. from Mukesh Patel School of Technology, Management and
Engg., SVKM's NMIMS University, Vile-Parle (W), Mumbai, INDIA. She has more than 11 years of experience in teaching. Currently working as Assistant Professor in Dept. of Computer Engineering at Thadomal Shahani Engineering College, Mumbai. She is member of International Association of Engineers (IAENG) and International Association of Computer Science and Information Technology (IACSIT). Her areas of interest are Image Processing, Signal Processing and Computer Graphics. She has 80 papers in National /International Conferences/journal to her credit.

Sanjay Ramkrishna Sange received B.E. in Industrial Electronics degree in 1994 from Amravati University, Maharashtra and M.E. in Computer Engg. from Mumbai University, Mumbai, Maharashtra in 2008. His research area is Image Processing. $\mathrm{He}$ is presently Assistant Professor and pursuing his $\mathrm{PhD}$. from SVKM's NMIMS University, MPSTME, Mumbai.

He has presented 5 National, 5 International papers and two Journal papers. His 3 papers are uploaded on Springer link and for one paper he has got "Best Paper" award in 2010. One paper is published in IEEE Transaction Digital CD Library in 2009. One paper is published online for International Journal of Computer Applications (IJCA). 